\title{
Exosomal miR-130b-3p targets SIK1 to inhibit medulloblastoma tumorigenesis
}

\author{
Saihua Huang ${ }^{1}$, Ping Xue ${ }^{1,2}$, Xiao Han ${ }^{1}$, Caiyan Zhang ${ }^{1}$, Lan Yang ${ }^{1}$, Lijuan Liu' ${ }^{1}$ Xiang Wang ${ }^{1}$, Hao Li ${ }^{2}$, Jinrong Fu and \\ Yufeng Zhou (D) ${ }^{1,3}$
}

\begin{abstract}
Exosomes are an important carrier for cell communication. miRNAs in exosomes are potential biomarkers and therapeutic targets in different types of cancer. However, the role of exosomal miRNAs in medulloblastoma (MB) patients is largely unknown. In this study, we reported that there was a higher level of miR-130b-3p in exosomes derived from MB patient plasma compared with exosomes from healthy control plasma. Exosomes from MB patient plasma could transfer miR-130b-3p to an MB cell line and played suppressor roles for cell proliferation. miR-130b-3p suppressed MB tumorigenesis by targeting a previously unknown target, serine/threonine-protein kinase 1 (SIK1), through the p53 signaling pathways. In addition, we found an unreported role of SIK1 in promoting MB tumor growth and an SIK1 inhibitor could inhibit MB cell proliferation. This research provides new insight into the molecular mechanism of $\mathrm{MB}$ and may provide a new therapeutic strategy for MB treatment.
\end{abstract}

\section{Introduction}

Medulloblastoma (MB) is the most common malignant brain tumor in children, accounting for $20-30 \%$ of children with central nervous system malignancies ${ }^{1}$, and the peak incidence is between 3 and 4 years and 8 and 9 years of age ${ }^{2}$. The present clinical treatments for MB involve a combination of surgery, cranio-spinal radiotherapy (in children $\geq 3$ years of age) and chemotherapy, but these treatments are often associated with significant side effects $^{3-5}$. Major challenges for patients and physicians include a lack of early diagnostic biomarkers, the strong tendency of this cancer to metastasize along the cerebrospinal axis, and a lack of precision treatment options based on the tumor molecular background ${ }^{6,7}$. Therefore,

\footnotetext{
Correspondence: Jinrong Fu (fujinrong@hotmail.com) or

Yufeng Zhou (yfzhou1@fudan.edu.cn)

IInstitute of Pediatrics, Children's Hospital of Fudan University, and the

Shanghai Key Laboratory of Medical Epigenetics, International Co-laboratory of

Medical Epigenetics and Metabolism, Ministry of Science and Technology,

Institutes of Biomedical Sciences, Fudan University, 200032 Shanghai, China

${ }^{2}$ Department of Neurosurgery, Children's Hospital of Fudan University,

Shanghai, China

Full list of author information is available at the end of the article

These authors contributed equally: Saihua Huang, Ping Xue, Xiao Han

Edited by A. Stephanou
}

early diagnostic biomarkers, a comprehensive understanding of the molecular mechanisms involved in $\mathrm{MB}$ tumorigenesis, and methods to regulate these are urgently needed.

Exosomes are secreted by all living cells, and are small membrane vesicles of $50-150 \mathrm{~nm}$ in diameter containing microRNAs (miRNAs), protein, lipids, DNA and mRNA $^{8,9}$. Exosomes are an important carrier in cell communication ${ }^{10}$. miRNAs are highly conserved, singlestranded RNA molecules of 19-24 nucleotides long ${ }^{11}$. They regulate genes by binding to the $3^{\prime}$ untranslated region ( $3^{\prime} \mathrm{UTR}$ ) of target messenger RNA ${ }^{12,13}$. Exosomal miRNAs are emerging as novel regulators of cellular function $^{14-16}$. Exosomal miRNAs moving from cancer cells to endothelial cells could promote tumor metastasis ${ }^{17}$. Exosomal miRNAs secreted from immune cells were shown to exert an antiproliferative effect on tumor cells ${ }^{14}$. Therefore, miRNAs in exosomes are potential biomarkers and therapeutic targets in different types of cancer.

It has been reported that the miR-130 family plays an important role in the development of cancer, but the results vary in different cancers ${ }^{18-20}$. Yeung et al. ${ }^{21}$

\section{(c) The Author(s) 2020}

(c) (i) Open Access This article is licensed under a Creative Commons Attribution 4.0 International License, which permits use, sharing, adaptation, distribution and reproduction cc) in any medium or format, as long as you give appropriate credit to the original author(s) and the source, provide a link to the Creative Commons license, and indicate if changes were made. The images or other third party material in this article are included in the article's Creative Commons license, unless indicated otherwise in a credit line to the material. If material is not included in the article's Creative Commons license and your intended use is not permitted by statutory regulation or exceeds the permitted use, you will need to obtain permission directly from the copyright holder. To view a copy of this license, visit http://creativecommons.org/licenses/by/4.0/. 
showed that miR-93/miR-130b could promote tumor growth by inhibiting Tp53INP1 expression. However, it has been found that miR-130b-3p could directly target the Notch ligand Delta-like 1 (DLL1) and inhibit breast carcinoma cell invasion and migration ${ }^{22}$. It has been suggested that the upregulation of miR-130b-3p contributed to the development of thyroid adenomas by targeting the CCDC6 gene ${ }^{23}$. In a pilot RNA-seq analysis of exosome in the plasma of MB patients (GSE123376), we found exosomal miR-130b-3p was elevated in the plasma of $M B$ patients. However, the effects of miR-130b-3p derived from exosomes in $\mathrm{MB}$ remain unknown. In the current study, we confirmed that there was a higher level of miR$130 \mathrm{~b}-3 \mathrm{p}$ in exosomes derived from the MB patient plasma than in those from healthy control plasma. We investigated the tumor suppressor role of miR-130b-3p in MB in vitro and in vivo by targeting a previously unknown target, serine/threonine-protein kinase 1 (SIK1), through the p53 pathways. Our research provides new insights into the molecular mechanism of $\mathrm{MB}$ and may offer new therapeutic strategies for MB treatment.

\section{Materials and methods}

\section{Patients and samples}

Blood samples from MB patients and healthy donors were obtained from Children's Hospital of Fudan University. The study protocol was approved by the hospital institutional review board and written informed consent was obtained from each participant. Blood plasma was retrieved from whole-blood samples $(4 \mathrm{~mL})$ via centrifugation at $400 \times g$ for $10 \mathrm{~min}$ at $4{ }^{\circ} \mathrm{C}$ and aliquoted and stored at $-80^{\circ} \mathrm{C}$ until analysis. The relevant characteristics of patients are summarized in Supplementary Table 1.

\section{Cell lines and cell culture}

The human MB cell line, Daoy, was purchased from the Shanghai Institute of Cell Biology, Shanghai, China. The cells were cultured in MEM (Minimum Essential Medium) supplemented with $10 \%$ fetal bovine serum (FBS), 1\% sodium pyruvate, nonessential amino acids and penicillin-streptomycin. The D283 Med cells were cultured in RPMI 1640 media. Cells were maintained at $37^{\circ} \mathrm{C}$ in a humidified atmosphere containing $5 \% \mathrm{CO}_{2}$.

THP-1 monocytes were maintained in RPMI 1640 media supplemented with $10 \%$ heat-activated FBS, penicillin $(100 \mathrm{U} / \mathrm{mL})$ and streptomycin $(100 \mu \mathrm{M})$. HMO6 cells, an immortalized human microglial cell line, were cultured in DMEM (Dulbecco's Modified Eagle Medium) high-glucose medium. For exosome collection, THP-1 cells $\left(1 \times 10^{7}\right)$ were plated in $10 \mathrm{~cm}$ dishes with complete culture media overnight, and transfected with miR-130b$3 \mathrm{p}$ mimic or NC. At $48 \mathrm{~h}$, washed twice with PBS, and media was replaced with FBS free media and incubated overnight. Media was collected at $24 \mathrm{~h}$ for exosome purification. For exosome purification, HMO6 cells were cultured as mentioned above for THP-1 cells.

\section{Exosome isolation, identification and labeling}

Exosomes were isolated from the plasma of healthy control subjects, $\mathrm{MB}$ patients and culture supernatant of THP-1 or HMO6 cells with ultracentrifugation method by series of centrifugation at $4{ }^{\circ} \mathrm{C}: 300 \times g$ for $10 \mathrm{~min}$, $2000 \times g$ for $10 \mathrm{~min}$ to remove cellular debris and large apoptotic bodies, $100,000 \times g$ for $70 \mathrm{~min}$ to precipitate exosomes, and $100,000 \times g$ for $70 \mathrm{~min}$ to obtain purer exosomes. Exosomes were quantified by a BCA Protein Assay Kit (Takara).

For transmission electron microscopy (TEM) analysis, isolated exosomes were fixed with $4 \%$ paraformaldehyde and $4 \%$ glutaraldehyde in $0.1 \mathrm{M}$ phosphate buffer ( $\mathrm{pH} 7.4)$ and then placed on a carbon-coated copper grid and immersed in a $2 \%$ phosphotungstic acid solution for examination (JEM-1200EX; JEOL Ltd., Japan). Western blot analysis was used to detect biomarkers of exosomes including CD9 and CD63, and the Golgi marker GM130 was used as a negative control. To observe the cellular uptake of exosomes, purified exosomes were labeled using a PKH67 labeling kit (Sigma-Aldrich). After co-culture with labeled exosomes for $12 \mathrm{~h}$, Daoy cells were fixed and stained using Hoechst33258. Images were obtained using a Lecia TCS-SP5 LSM.

\section{Treatment of Daoy and D283 with exosomes}

The Daoy and D283 cells were seeded into 24-well plates or 96 -well plates overnight, and then $50 \mu \mathrm{g} / \mathrm{mL}$ of exosomes secreted from the plasma of healthy control subjects or MB patients, THP-1-transfected miR-130b-3p mimic or NC was added into per well. After being incubated for $24 \mathrm{~h}$ at $37^{\circ} \mathrm{C}$, the cells were harvested for cell survival assays and RT-qPCR.

\section{Transfection}

The miR-130b-3p mimic, corresponding negative control, si-SIK1 and nonspecific siRNA negative control used herein were purchased from GenePharma (Shanghai, China). The transfection was conducted using Lipofectamine RNAiMAX (Invitrogen, Carlsbad, CA, USA) at a final concentration of $200 \mathrm{nM}$ in accordance with the manufacturer's instructions. The sequences of mimic and siRNA are listed in Supplementary Table 2.

\section{RNA isolation and real-time quantitative PCR}

Total RNA was extracted from the cells or tissues using TRIzol(Invitrogen, USA) in accordance with the manufacturer's protocol. For the expression level of exosomal miRNAs verification, commercial kit was used to extract total RNA from exosomes (exoRNeasy Serum/Plasma Maxi Kit (50), Qiagen). The expression of miRNAs was 
determined using TaqMan microRNA assay kits (ABI, USA) with U6 as an internal control. To analyze the mRNA levels of SIK1, total RNA was reverse transcribed with oligo dT primers using the PrimeScript RT Reagent kit (Takara, Dalian, China). Actin served as an internal control. Relative RNA expression levels were quantified using the $2^{-\Delta \Delta C T}$ method. The sequences for primers are listed in Supplementary Table 3.

\section{Cell growth assay}

To assay cell proliferation, the cell counting kit- 8 (CCK-8) colorimetric assay (DOJINDO Molecular Technologies, Inc., Kumamoto, Japan) was performed. Daoy cells were placed in 96-well plates and cultured in the complete medium. After incubation, the cells were transfected with $200 \mathrm{nM}$ of the miR-130b-3p mimic/miRNC or si-SIK1/si-NC. D283 Med cells were transfected with the lentiviral vector for delivery of miR-130b-3p and the corresponding negative control purchased from GenePharma (Shanghai, China). Daoy cells were treated using an inhibitor of SIK1 (HG-9-91-01) for $1 \mathrm{~h}$, and then tested using CCK-8 kit. Each sample was assayed in triplicate. Cell viability was determined at $0,24,48$ and $72 \mathrm{~h}$ using the CCK- 8 assay. The optical density of each well was assessed using a Microplate reader at $450 \mathrm{~nm}$ to determine cell viability. Each experiment was performed in triplicate.

\section{Colony-formation assay}

We also performed a colony-forming ability assay to assess Daoy cell proliferation. Followed by transfection with the miR-130b-3p mimic, si-SIK1, or NC, Daoy cells were seeded into $60 \mathrm{~mm}$ culture dishes at a density of 500 cells/dish and cultured in a $5 \% \mathrm{CO}_{2}$ incubator at $37^{\circ} \mathrm{C}$. After 2 weeks, the cells were stained using crystal violet.

\section{Cell apoptosis assays}

Daoy cells were cultured in six-well plates for $48 \mathrm{~h}$ after miRNA and siRNA transfection and D283 Med cells were transfected with the lentiviral vector for delivery of miR$130 \mathrm{~b}-3 \mathrm{p}$ and the corresponding negative control, and then treated using $100 \mu \mathrm{mol} \mathrm{H}_{2} \mathrm{O}_{2}$ overnight. The apoptosis assays were evaluated through flow cytometry using the FITC Annexin V Apoptosis Detection Kit I (BD, San Diego, CA, USA) in accordance with the manufacturer's instructions.

\section{Wound-healing assays}

For wound-healing assays, transfected MB cells were grown to $100 \%$ confluence in six-well plates, after which scratch-wounds were created in the monolayers using a sterile $200 \mu \mathrm{L}$ pipette tip. Photographs were taken using phase-contrast microscopy (Leica, Germany) immediately and $24 \mathrm{~h}$ after wound creation.

\section{Cell invasion assay}

Cell invasion assay was performed using Matrigelcoated transwell chambers $(8 \mu \mathrm{m}$ pore size; Costar). A total of $100 \mu \mathrm{L}$ transfected cell suspension $\left(2 \times 10^{4}\right.$ cells/ $\mathrm{mL}$ ) was added to the upper chamber of the transwell plates. A total of $500 \mu \mathrm{L}$ complete medium was added to the bottom chambers. After incubation for $24 \mathrm{~h}$ at $37^{\circ} \mathrm{C}$, nonmigrating cells were removed using cotton swabs. The cells that permeated the Matrigel-coated membrane and migrated to the bottom were fixed using paraformaldehyde and then stained using crystal violet.

\section{Luciferase reporter assay}

The SIK1 3'UTR, including wild-type miR-130b-3p binding sites, and the mutated SIK1 $3^{\prime}$ UTR were respectively cloned into the downstream of the firefly luciferase gene within the psiCHECK-2 vector (Promega). For reporter assays, HEK-293T cells were seeded in 96-well plates in triplicate and allowed to settle for $24 \mathrm{~h}$. The cells were then cotransfected with $10 \mathrm{ng}$ firefly luciferase reporter plasmid and an equal amount $(200 \mathrm{nM})$ of miR130b-3p mimic or scrambled negative control RNA using Lipofectamine 2000 (Invitrogen, Carlsbad, CA, USA). At $24 \mathrm{~h}$ post transfection, the cells were assayed using a luciferase assay kit (Promega, Madison, WI, USA).

\section{Western blotting assay}

Cells were washed in PBS and lysed using RIPA lysis buffer supplemented with a protease phosphatase inhibitor. Total protein was quantified using a BCA Protein Assay Kit (Takara), and equal amounts of whole-cell lysates were resolved through SDS-polyacrylamide gel electrophoresis and transferred to polyvinylidene difluoride membranes (Bio-Rad, USA). The blots were blocked in bovine serum albumin (5\% w/v in PBS $+0.1 \%$ Tween20) for $1 \mathrm{~h}$ at room temperature and immune stained using antibodies at $4{ }^{\circ} \mathrm{C}$ overnight. The membranes were incubated using primary antibodies and horseradish peroxidase-conjugated secondary antibodies. Enhanced chemiluminescence assays were used to detect the signal. The antibodies against CD9 (1:1000), GM130 (1:1000), p53 (1:1000), Bcl-2 (1:1000) were purchased from Cell Signaling Technology, Inc. Anti-CD63 (1:1000) and anti-SIK1 (1:1000) were purchased from Abcam.

\section{Vector constructs and lentiviral production}

The lentiviral vectors for delivery of miR-130b-3p and the corresponding negative control were purchased from GenePharma (Shanghai, China). sh-SIK1 was amplified and cloned into the pLenti vector after double digestion by BamHI and EcoRI to form sh-SIK1 pLenti vector and then the constructed vector was sequenced (Sangon Biotech, Shanghai, China). For stable transfection, cells were transfected with sh-SIK1 or negative control 
(sh-GFP) lentiviral particles, in accordance with the manufacturer's protocol. The Daoy cells were transfected in growth media containing $1 \mu \mathrm{g} / \mathrm{mL}$ puromycin and until the green fluorescent protein included in the plasmid vector was visualized. Total RNA in stably transfected cells was extracted to detect the efficiency of stable transfection.

\section{Animal experiments}

Male BALB/c nude mice (6 weeks old, obtained from the SLRC Laboratory Animal Center, Shanghai, China) were subcutaneously injected with $5 \times 10^{6}$ Daoy-miR130b-3p cells, Daoy control cells, sh-GFP cells and shSIK1 cells. The injected cells were suspended in $100 \mu \mathrm{L}$ of $\mathrm{PBS} /$ matrigel (v/v, 1:1). Tumor volumes were measured using calipers and calculated using the following formula: volume $=$ length $\times(\text { width })^{2} / 2$. The curve of tumor growth was drawn based on tumor volume and corresponding time (days) after treatment. When the animals were terminated, the tumor tissues were removed and weighed. The animal assay complied with the Guide for the Care and Use of Laboratory Animals and was approved by the Animal Studies Committee of the Children's Hospital of Fudan University.

\section{Immunohistochemistry, hematoxylin and eosin staining and fluorescence in situ hybridization}

Dissected tissues were formalin-fixed, paraffinembedded, sectioned and stained using hematoxylin and eosin in accordance with standard histopathological techniques. For immunohistochemistry (IHC), sections were incubated with primary antibodies against Ki67 and SIK1, and secondary antibodies, followed by staining and imaging.

The work flow of fluorescence in situ hybridization (FISH) (GenePharma, Shanghai, China) for miR-130b-3p is described below. In brief, paraffin sections were deparaffinized by xylene and gradient alcohol (100\%, 95\%, $90 \%, 80 \%, 70 \%)$. The slices were treated by Proteinase K and denaturation solution. Hybridization: (1) Probe dilution: Add DEPC water to the probe dry powder product to obtain a stock solution with a concentration of approximately $100 \mu$ M. (2) Preparation of probe mixed solution. (3) Prepare a wet box, place the slices horizontally, add $100 \mu \mathrm{L}$ of the denatured probe mixture to each slice and incubate at $37^{\circ} \mathrm{C}$ for $12-16 \mathrm{~h}$. Stringency wash: This step is essential for removing the overload probes and nonspecific probe binding. The slices were incubated by DAPI for nuclear staining.

\section{Statistical analysis}

All data are shown as the means \pm SEM. Student's $t$ test was used for comparisons between two groups, and oneway analysis of variance was used for multiple comparisons. $p<0.05$ was considered to indicate statistical significance $\left(" p<0.05,{ }^{* * *} p<0.01,{ }^{* * * *} p<0.001\right)$.

\section{Results}

miR-130b-3p is upregulated in exosomes derived from MB patient plasma and can be transferred to tumor cells through exosomes

To identify differentially expressed exosomal miRNA in $\mathrm{MB}$, we previously analyzed the miRNA expression profiles of exosomes derived from $\mathrm{MB}$ patient plasma by RNA-seq (GSE123376, submitted in other draft), and found miR-130b-3p was highly expressed in exosomes from MB patient plasma compared with healthy controls $(n=3$, fold-change $>1.5)$ (Supplementary Fig. S1). Subsequently, we evaluated the expression of exosomal miR130b-3p derived from MB patients and healthy control plasma in a larger population of samples. Exosomal fractions were prepared from $\mathrm{MB}$ patient plasma by ultracentrifugation. TEM revealed that the exosomes showed typically round shapes, ranging in size between 50 and $150 \mathrm{~nm}$ (Fig. 1a). To confirm the identity of the isolated exosomes, we examined some specific exosome markers, CD9 and CD63. As shown in Fig. 1b, CD9 and CD63 were highly enriched in the isolated exosomes. Conversely, the negative maker of GM130 (Golgi marker) was almost undetectable in the isolated exosomes, but highly expressed in cells (Fig. 1b). It was found the expression of exosomal miR-130b-3p in MB patients was significantly higher than that of control (Fig. 1c). We also detected the expression of miR-130b-3p in the peripheral blood mononuclear cells (PBMCs) from MB patients and healthy controls by RT-qPCR, and found that the expression of miR-130b-3p in the PBMCs of MB patients was notably higher than that of healthy control (Fig. 1d). We further evaluated the expression of miR-130b-3p in $\mathrm{MB}$ tissues and adjacent noncancerous tissues (the clinical characteristics of the patients are shown in Supplementary Table 1), and found that the expression level of miR130b-3p was significantly lower in tumor tissues than in nontumor tissues (Supplementary Fig. S2a).

It was reported exosomes played very important roles in communication between cells, and miRNAs were reported to be responsible for these roles of exosomes. As mentioned before, miR-130 family plays important roles in tumorigenesis; we thus hypothesized that exosomal miR-130b-3p in plasma could transfer into MB cells. Exosomes labeled with a fluorescent dye, PKH67, were added to Daoy culture for $12 \mathrm{~h}$. After incubation, fluorescence microscope imaging showed the presence of PKH67 spots in recipient Daoy cells, suggesting that labeled exosomes were delivered to Daoy cells (Fig. 1e). We assessed the expression of miR-130b-3p in Daoy cells that were cocultured with exosomes derived from plasma. The expression of miR-130b-3p was higher in cocultured 
A

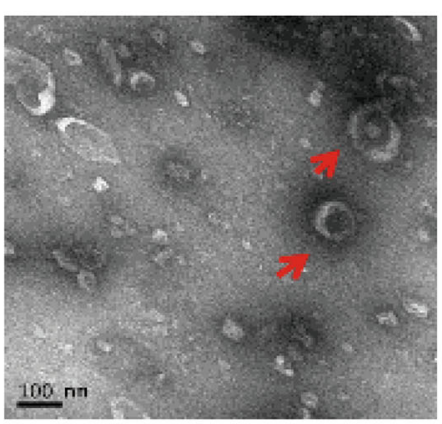

C

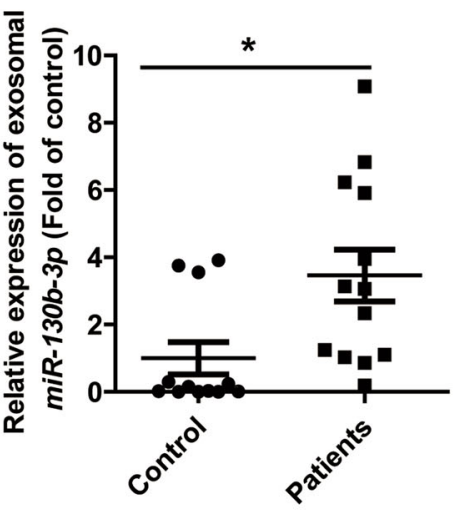

B

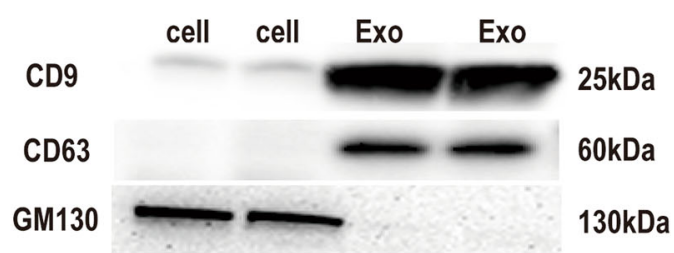

E

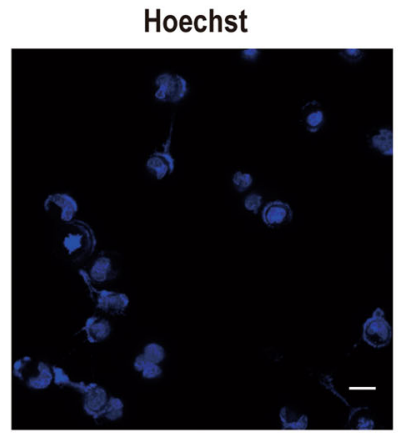

F

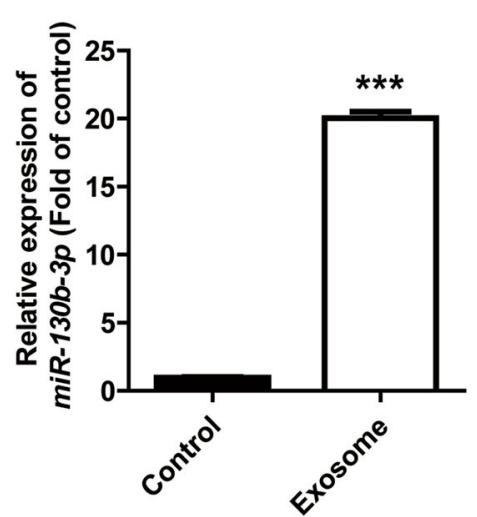

PKH67

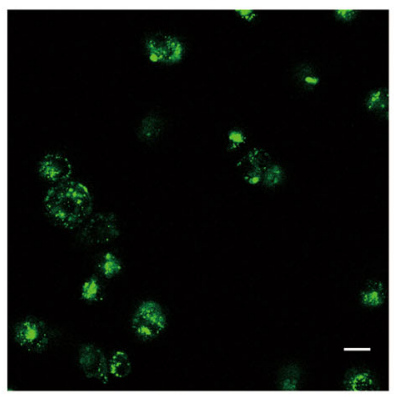

Merge
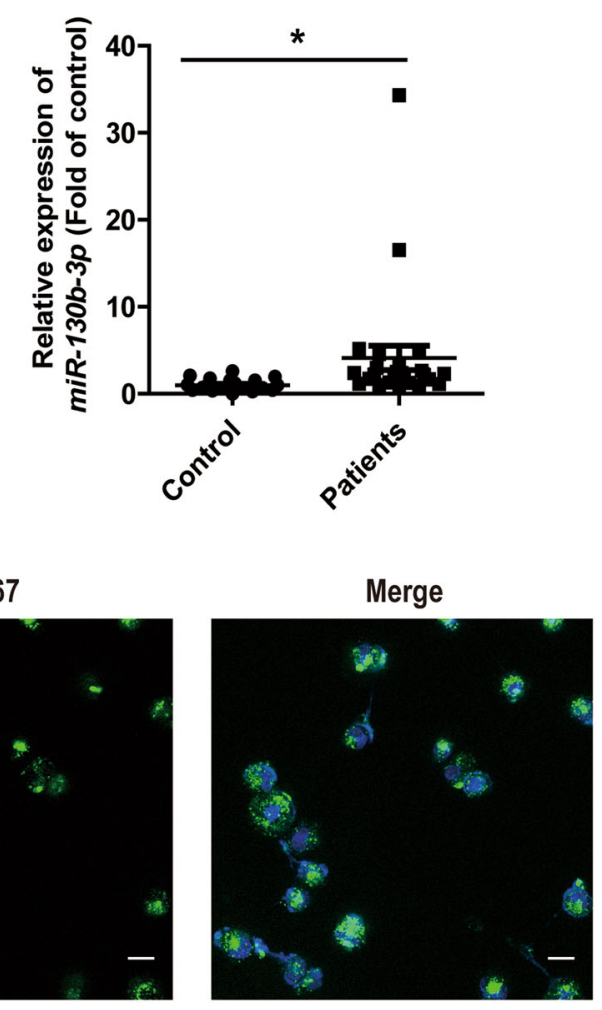

Fig. 1 (See legend on next page.) 
(see figure on previous page)

Fig. 1 miR-130b-3p can be transferred to tumor cells through exosomes. a The exosomes was assessed using transmission electron microscopy. Scale bar, $100 \mathrm{~nm}$. b Western blot analysis of the exosomal marker, CD9 and CD63; Golgi marker, GM130. c Exosomal miR-130b-3p expression in healthy and MB patient plasma detected by RT-qPCR. $\mathbf{d}$ The expression of miR-130b-3p in healthy and MB patient PBMCs detected by RT-qPCR. e Exosomes were isolated from MB patient plasma, dyed with PKH67 (green) and cocultured with Daoy cells for $12 \mathrm{~h}$, then dyed with Hoechst33258 (blue) and viewed with confocal microscopy (original magnifications $\times 200$ ). $\mathbf{f}$ The expression of miR-130b-3p in Daoy cells cocultured with the exosomes isolated from MB patient plasma, detected by RT-qPCR. Data represent means \pm SEMs from three independent experiments. ${ }^{*} p<0.05$, ${ }^{* * *} p<0.001$.

with exosomes isolated from the MB patient plasma (Fig. 1f). Therefore, the data showed that exosomes from MB patient plasma could transfer miR-130b-3p into tumor cells.

\section{Exosomal miR-130b-3p inhibit MB cell proliferation in vitro}

To identify the effect of exosomal miR-130b-3p on MB proliferation, we first extracted exosomes from the MB patient plasma and healthy control, which were used to treat the Daoy and D283. We tested the expression of miR-130b-3p in Daoy and D283 that were cocultured with different concentrations of exosomes derived from MB patient plasma by RT-qPCR. The expression of miR$130 \mathrm{~b}-3 \mathrm{p}$ was the highest in these cells which were cocultured with $50 \mu \mathrm{g} / \mathrm{mL}$ exosomes (Fig. 2a, b). It was found that there was a significantly lower proliferation rate when Daoy and D283 incubated with exosomes derived from $\mathrm{MB}$ patient plasma than with control (Fig. 2c, d).

In our study, we found miR-130b-3p was upregulated in the plasma exosome and PBMCs while it was downregulated in tumor sites (Fig. 1c, d; Supplementary Fig. S2a). A possible reason for this phenomenon was that exosomal miR-130b-3p in the plasma might be derived from monocytes or macrophages rather than tumor cells. Exosomes derived from macrophage account for a large proportion of circulating microvesicles in blood ${ }^{24}$. As THP-1 (a human leukemia monocytic cell line) could be readily differentiated into macrophages, and released high levels of exosomes, we used the THP-1 monocyte-derived macrophages as a model cell line for generation of immune cell exosomes ${ }^{25}$. We extracted the exosomes from THP-1-transfected with miR-130b-3p mimic or NC, then cocultured with Daoy and found that exosomes from THP-1 cells could also transfer into MB cells, and the expression of miR-130b-3p was higher in cocultured with exosomes derived from THP-1 (Fig. 2e, f). After coculturing of Daoy or D283 with the exosomes of THP-1, we found that the cell proliferation was inhibited by exosomes from miR-130b-3p-overexpressed THP-1 cells (Fig. 2g, h). To further confirm that this phenomenon was caused by exosomes, we used the neutral sphingomyelinase inhibitor GW4869 to treat THP-1 overexpressing miR-130b-3p to inhibit exosome secretion. The tumor cell proliferation was inhibited when the cells were cocultured with the miR-130b-3p-overexpressed THP-1 medium, while there was no change in the GW4869treated medium (Fig. 2i, j). These data indicated that the effects on cell proliferation were at least partly due to the presence of exosomes, and that these exosomes from THP-1 could be eliminated by GW4869 treatment. Same as THP-1, we found HMO6 (a type of glial cell, equivalent to a macrophage in the brain) could secret exosomes containing miR-130b-3p (Supplementary Fig. S3a, b). We also found there was higher number of CD68 + cells in the MB tissues (Supplementary Fig. S3c). Together, these data showed that miR-130b-3p could be passed by exosomes, and exosomal miR-130b-3p reduced cell growth.

\section{miR-130b-3p functions as a tumor suppressor for MB cells in vitro}

To investigate the potential biological functions of intracellular miR-130b-3p in MB, we performed gain-offunction analysis using miR-130b-3p mimic to assess its role in cell proliferation and apoptosis. The overexpression of miR-130b-3p in Daoy and D283 Med cells, achieved through transfection with miR-130b-3p mimic, inhibited cell growth (Fig. 3a) and colony formation (Supplementary Fig. S4a). In addition, miR-130b-3p mimic markedly increased the rate of apoptosis in Daoy and D283 Med cells compared with the negative control (Fig. 3b, c). We also examined the effects of miR-130b-3p on cell migration ability by performing a wound-healing assay. As D283 Med cells are suspension cells, we did not detect the motility. Daoy cells with miR-130b-3p overexpression showed a decreased ability to close the wound compared with cells transfected with negative control mimic, suggesting that miR-130b-3p inhibited cell migration (Fig. 3d). In addition, the effect of miR-130b-3p on cell invasion, a hallmark of malignancy, was examined using a transwell assay. miR-130b-3p significantly reduced cell invasion of Daoy cells by $52 \%$ (Fig. 3e). Therefore, miR-130b-3p considerably suppressed the migration and invasiveness of $\mathrm{MB}$ cells.

\section{SIK1 is a target of miR-130b-3p}

MicroRNAs often target the $3^{\prime} U T R s$ of mRNAs to regulate their stability and translation efficiency. Using 


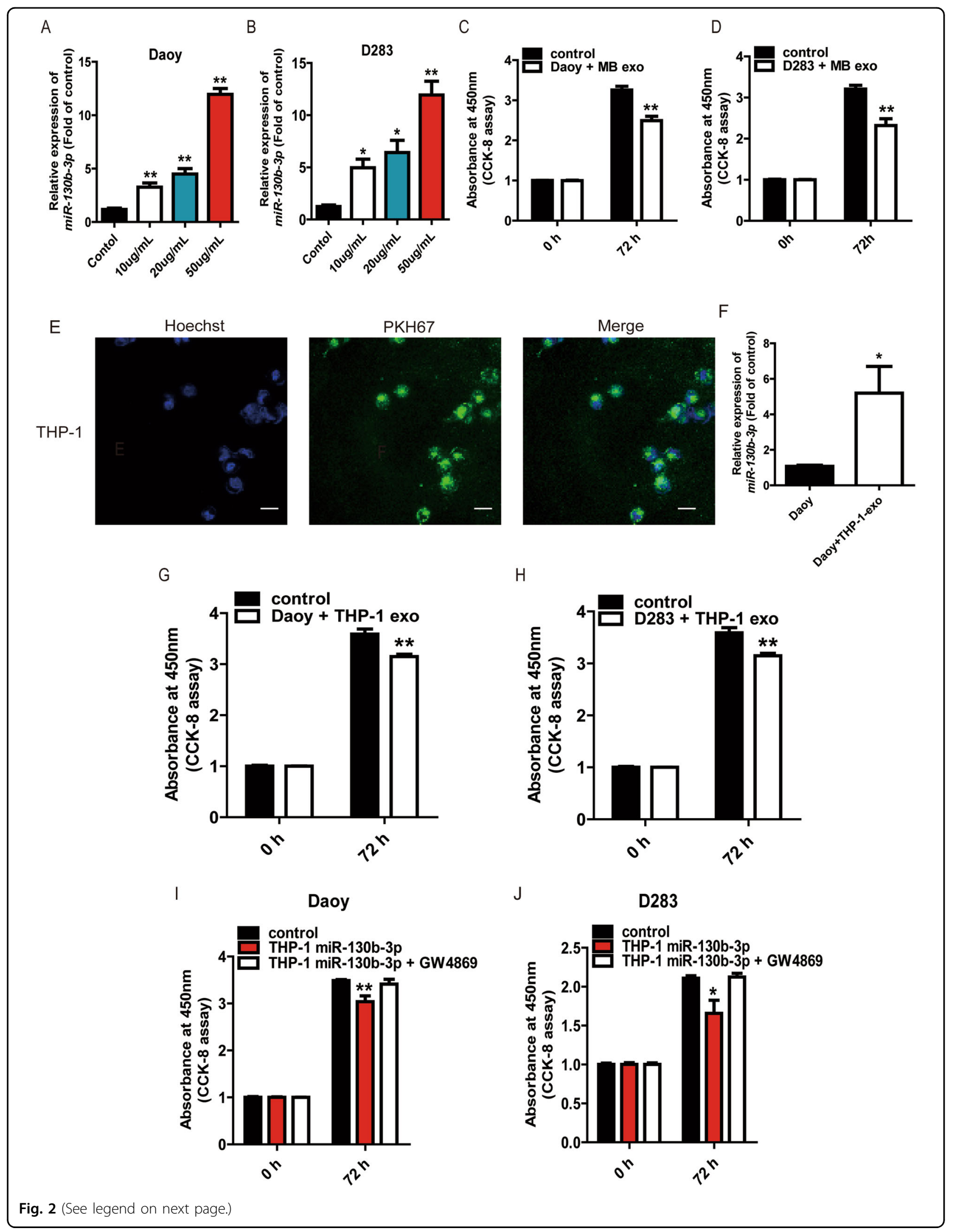


(see figure on previous page)

Fig. 2 Exosomal miR-130b-3p inhibit MB cell proliferation in vitro. a, b RT-qPCR assay was used to detect the expression of miR-130b-3p in Daoy and D283 cocultured with the different concentrations of exosomes $(10,20,50 \mu \mathrm{g} / \mathrm{mL})$ collected from MB patient plasma. $\mathbf{c}$, $\mathbf{d}$ The proliferative ability of Daoy and D283 treated with the different concentrations of exosomes derived from MB patient plasma by CCK-8. e Exosomes were isolated from culture supernatant from THP-1, dyed with PKH67 (green) and cocultured with Daoy, then dyed with Hoechst33258 (blue) and viewed with confocal microscopy (original magnifications $\times 200$ ). $\mathbf{f}$ The expression of miR-130b-3p in Daoy cocultured with the exosomes collected from THP-1 culture supernatant, detected by RT-qPCR. The proliferative ability of Daoy $(\mathbf{g})$ and D283 (h) treated with the exosomes derived from supernatant of THP-1 transfected with miR-130b-3p mimic or NC was tested by CCK-8. Cell proliferation of Daoy (i) and D283 (j) treated with supernatant of THP-1 transfected with miR-130b-3p mimic or NC and GW4869 was assessed by CCK-8. Data are presented as mean \pm SEM. ${ }^{*} p<0.05,{ }^{* *} p<0.01$.

two different target predicting algorithms including Target Scan and RNA22, 1506 mRNAs were predicted to be the target of miR-130b-3p (Fig. 4a). LKB1 (also known as Stk11) is a serine/threonine protein kinase that regulates growth and metabolism through adenosine monophosphate-activated protein kinase (AMPK) and 12 other closely related kinases. Studies have reported that LKB1 signaling plays important roles in cell proliferation and migration. There are 42 genes involved in LKB1 signaling events. We overlapped the Target Scan, RNA22 and LKB1 signaling events to get six candidate genes (SIK1, SIK3, ESR1, SMAD4, MAP2 and TSC1) (Fig. 4a). Next, we compared the mRNA levels of these genes in Daoy cells transfected with miR-130b-3p mimic and found that SIK1 was significantly downregulated among these six genes (Fig. 4b). To further investigate whether the SIK1 gene is a direct target of miR-130b-3p in $\mathrm{MB}$ cells, a dual luciferase reporter assay was performed using a reporter vector (psicheck2). Luminescence was measured after cotransfection with miRNA mimic and plasmid vectors in HEK293T cells. After cotransfection for $24 \mathrm{~h}$, the negative control miRNA did not decrease the luciferase activity. At the same time, transfection with the miR-130b-3p mimic did not reduce the luciferase activity produced by the negative control vector without the SIK1 3'UTR sequence. However, cells transfected with miR-130b-3p mimic and the SIK1 $3^{\prime}$ UTR vector showed markedly lower luciferase activity compared with the control and the negative control. In contrast, cotransfection of miR-130b-3p with the SIK13'UTR mutant showed no remarkable change in luciferase activity, indicating the direct binding between miR-130b-3p and the SIK1 3'UTR (Fig. 4c). The western blot analyses confirmed that miR-130b-3p also suppressed the endogenous expression of SIK1 at the protein level in MB cells (Fig. 4d). This suggests that SIK1 is a target of miR-130b-3p. Next, we analyzed SIK1 expression levels in $25 \mathrm{MB}$ tumor tissue specimens and 15 nontumor tissues through RT-qPCR, and the results showed that SIK1 mRNA expression levels were remarkably upregulated in the $\mathrm{MB}$ tissue specimens compared with the nontumor tissues (Supplementary Fig. S2b).

\section{miR-130b-3p suppresses MB cell tumorigenesis through SIK1}

To test whether SIK1 was responsible for the reduced cell viability after miR-130b-3p mimic treatment, we measured cell proliferation following treatment using the SIK1 inhibitor HG-9-91-01. We found that HG-9-91-01 could significantly reduce cell proliferation, and this suggested that SIK1 itself could promote cell viability (Fig. 5a). Then, we knocked down SIK1 gene expression in Daoy cells using specific siRNAs. siSIK1-1641 was selected for the following experiments because of its significant knockdown efficiency (Fig. 5b). The suppression of SIK1 markedly decreased cell viability (Fig. 5c; Supplementary Fig. S4b) and increased annexin V-positive cell populations in Daoy cells (Fig. 5d). Therefore, the results from both the cell viability assay and the apoptosis assay upon si-SIK1 treatment were generally consistent with the results of the miR-130b-3p mimic treatment.

We also tested if SIK1 would affect cell motility. Daoy cells were transfected with si-SIK1 or NC. Twenty-fourhour wound-healing assay revealed that si-SIK1 effectively suppressed the migration capacity of Daoy cells compared with NC treatment (Fig. 5e). The transwell assay showed that si-SIK1 could reduce Daoy cell invasion (Fig. 5f). In conclusion, these results indicate that SIK1 promoted cell motility in $\mathrm{MB}$ cells in vitro and miR-130b-3p could reduce cell viability, promote cell apoptosis and inhibit cell motility in an MB cell line by targeting SIK1.

\section{miR-130b-3p promotes the activity of the p53 signaling pathways through downregulation of SIK1}

We further investigated the mechanism through which miR-130b-3p regulates cell apoptosis in MB. The p53 pathways were reported to be involved in the LKB1 pathway and are important in cell apoptosis. We speculated that miR-130b-3p-SIK1 promoted apoptosis via the p53 signaling pathways. In Daoy cells, p53 and BAX were upregulated by miR-130b-3p overexpression and SIK1 knockdown, while Bcl-2 was downregulated (Fig. 6a-e). Therefore, these findings suggest that miR$130 \mathrm{~b}-3 \mathrm{p}$ promotes the activity of the p53 signaling pathways through downregulation of SIK1 to regulate MB cell apoptosis. 

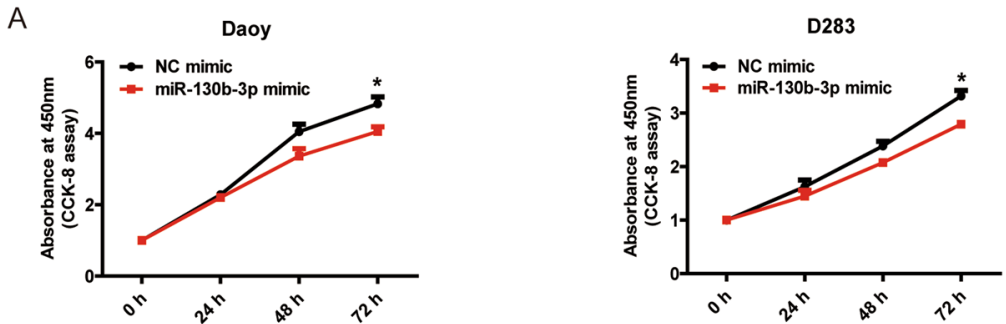

B

Daoy
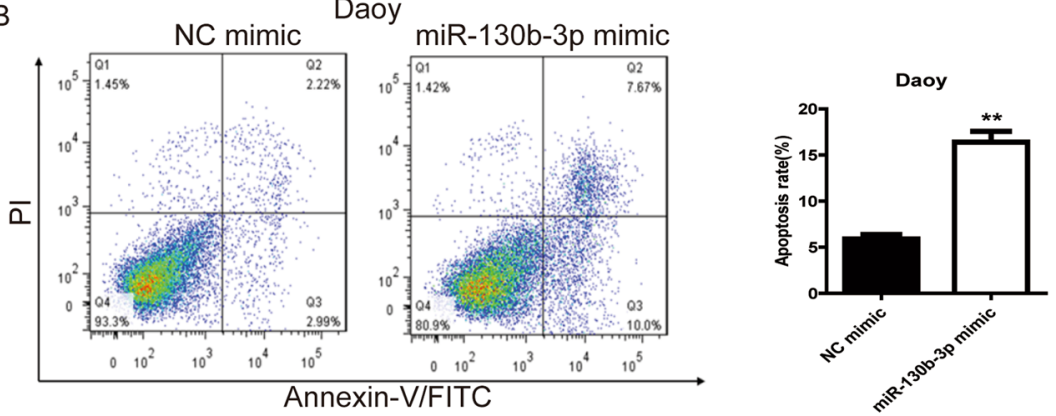

C

D283
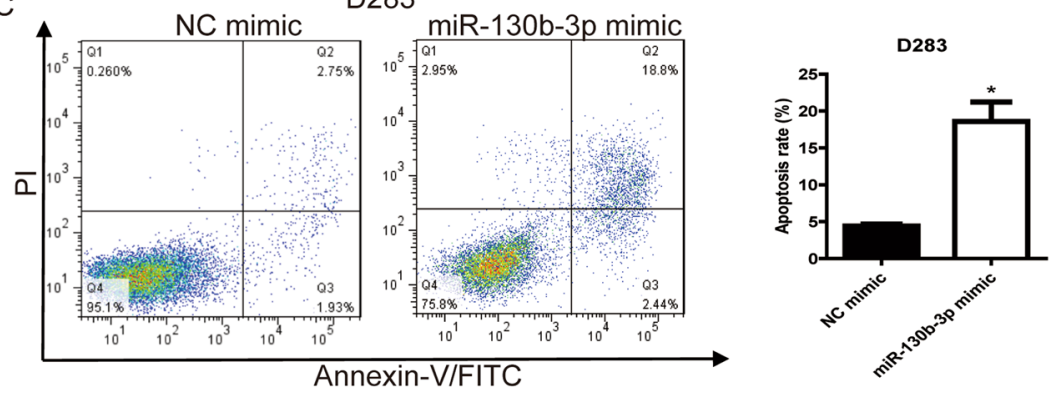

D
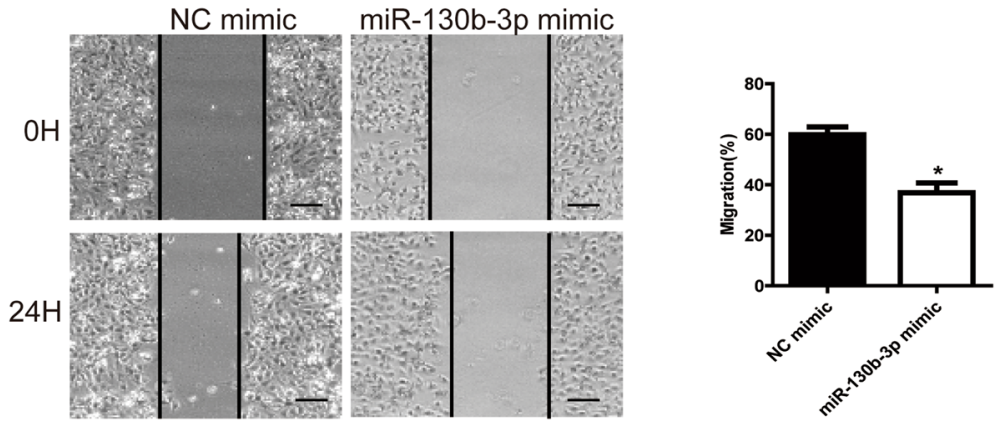

$\mathrm{E}$

NC mimic

miR-130b-3p mimic
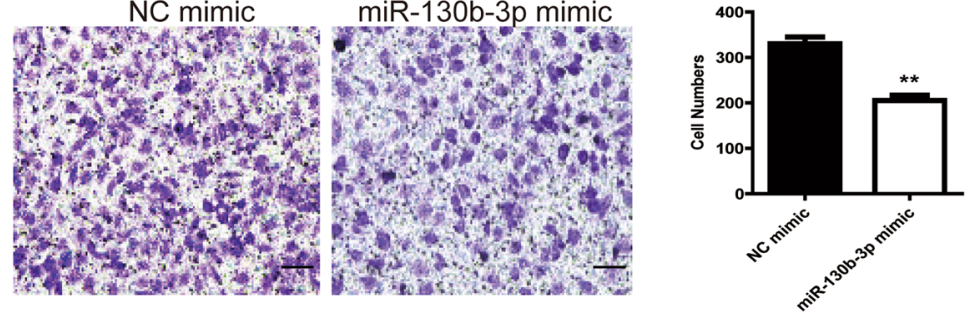

Fig. 3 miR-130b-3p functions as a tumor suppressor of MB cells in vitro. a The proliferative ability of MB cells transfected with miR-130b-3p mimic or NC was tested through CCK-8. b, c Cell apoptosis was detected through flow cytometry in MB cells transfected with miR-130b-3p mimic or negative control. $\mathbf{d}$ Cell migration was detected through a wound-healing assay in MB cells transfected with miR-130b-3p or NC (original magnifications $\times 200$ ). e Cell invasion analysis was performed through transwell invasion assays in MB cells transfected with miR-130b-3p or NC (original magnifications $\times 200$ ). Data represent means \pm SEMs from three independent experiments. ${ }^{*} p<0.05$, ${ }^{* *} p<0.01$. 
A

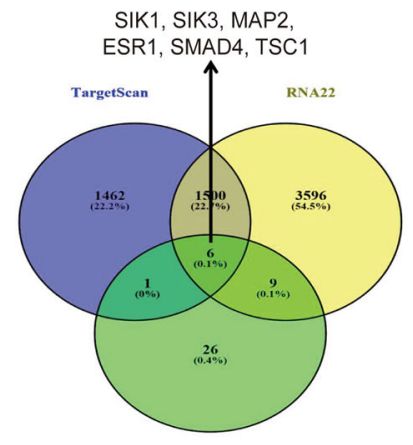

B

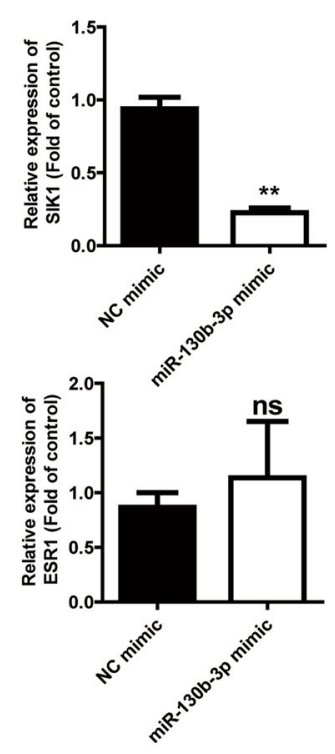

C

miR-130b-3p: 3'- UACGGGAAAGUAGUAACGUGAC - 5' ||| |||||

SIK1-3'-UTR: 5'- UGUUUUAUUUUUUUCUUGCACUGG - 3' SIK1-3'-mUTR: 5'- UGUUUUAUUUUUUUCAUCCUCĂG - 3'

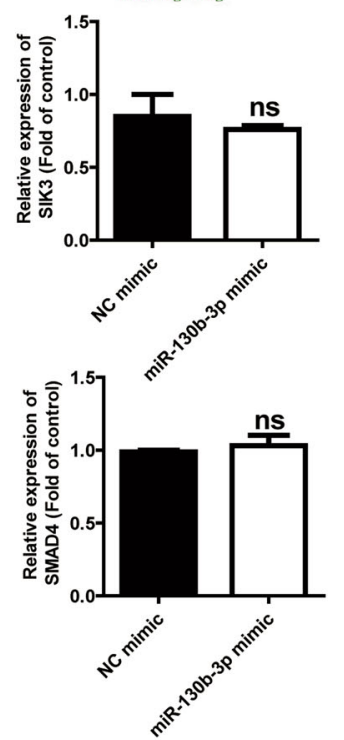

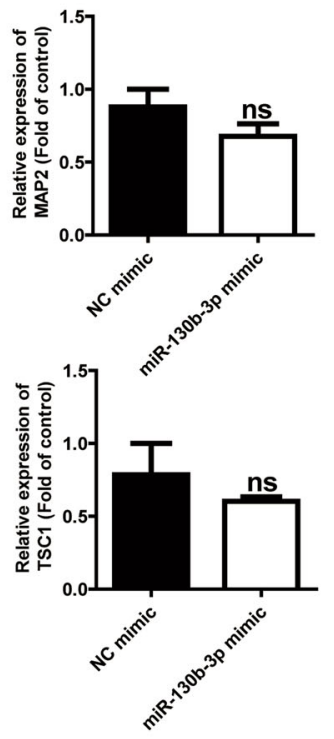

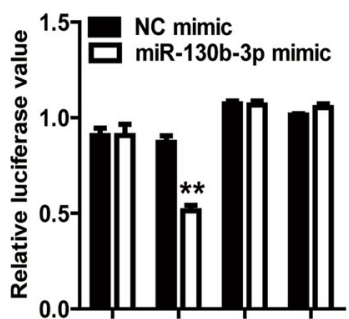

D
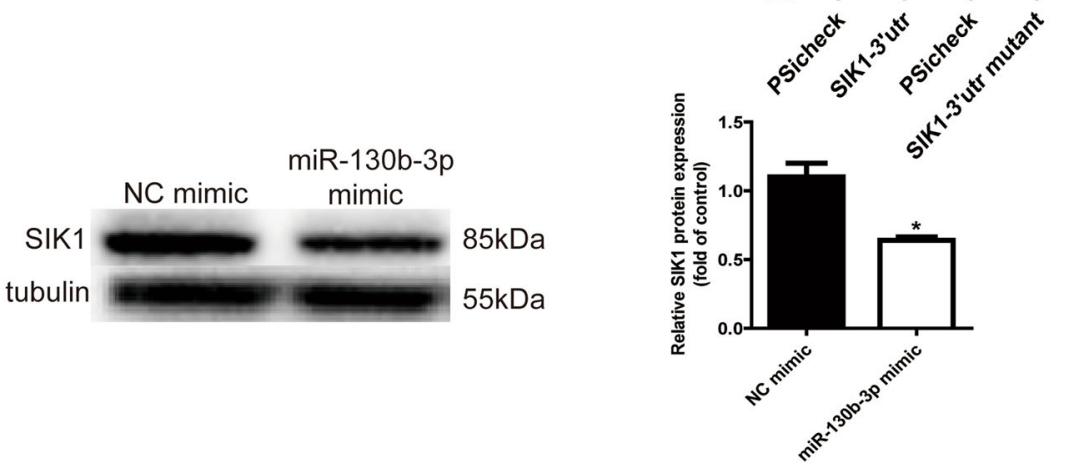

Fig. 4 SIK1 is a target of miR-130b-3p. a Venn diagrams showing the number of potential miR-130b-3p targets. b RT-qPCR analysis of six potential miR-130b-3p target genes in MB cells with miR-130b-3p overexpressed. c The potential binding sites of miR-130b-3p within the SIK1 mRNA 3'-UTR and 3'-mUTR. Luciferase reporter gene assays were used to analyze the effect of miR-130b-3p on luciferase activity. $\mathbf{d}$ SIK1 protein levels were detected through western blotting in MB cells transfected with miR-130b-3p mimic. NC negative control. Data represent means \pm SEMs from three independent experiments. ${ }^{*} p<0.05,{ }^{* *} p<0.01$. 


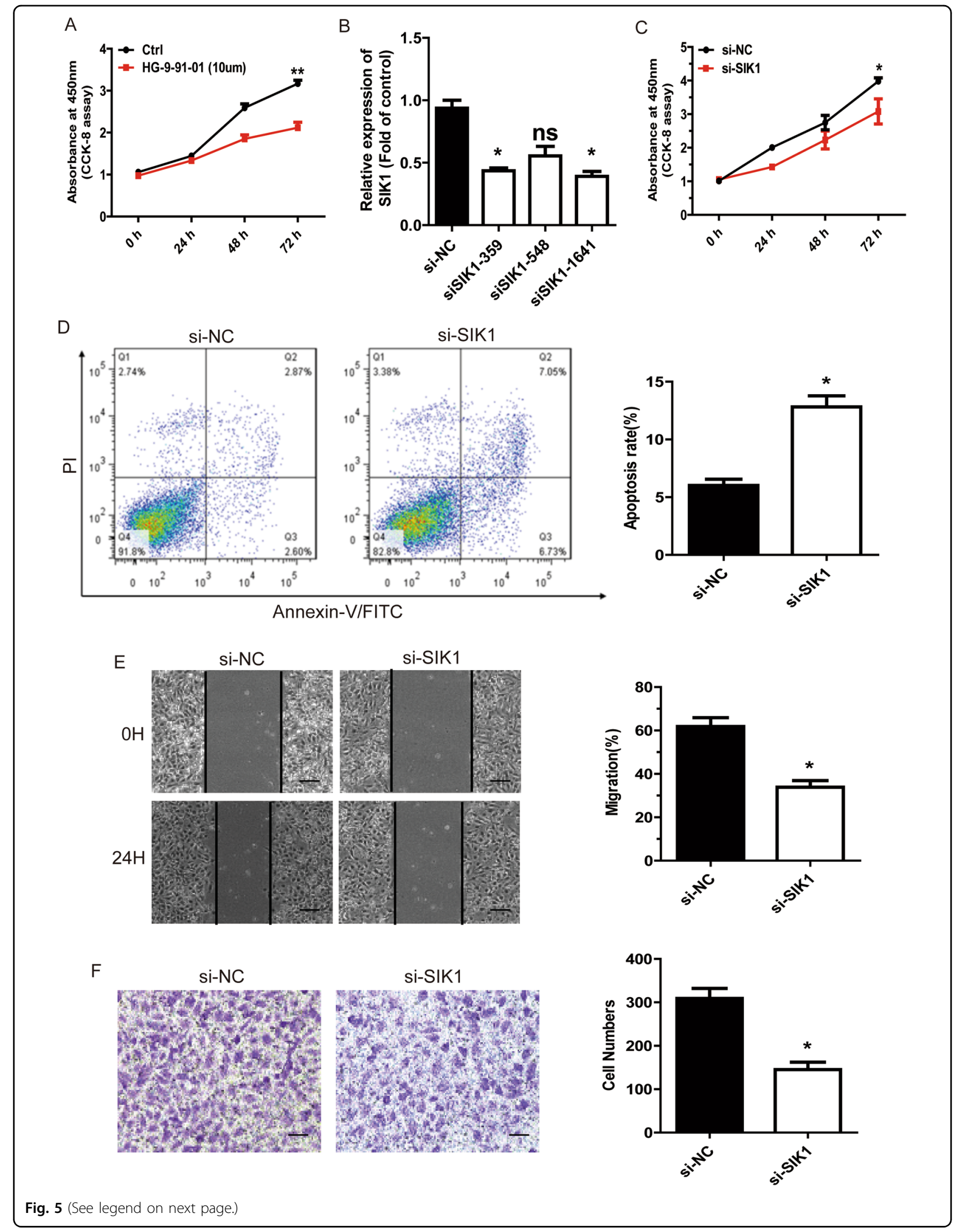


(see figure on previous page)

Fig. 5 miR-130b-3p suppresses MB cell tumorigenesis through SIK1. a CCK-8 assay in MB cells treated with SIK1 inhibitor. $\mathbf{b}$ The efficiency of siSIK1 knockdown was detected by RT-qPCR. c The proliferative ability of MB cells transfected with si-SIK1 or NC was tested through CCK-8. $\mathbf{d}$ Cell apoptosis was detected through flow cytometry in MB cells treated with si-SIK1 or negative control. e Cell migration was detected through woundhealing assay in MB cells transfected with si-SIK1 (original magnifications $\times 200$ ). $\mathbf{f}$ Cell invasion analysis was performed through transwell invasion assay in MB cells transfected with si-SIK1 (original magnifications $\times 200$ ). Data represent means \pm SEMs from three independent experiments. ${ }^{*} p<$ $0.05,{ }^{* *} p<0.01$.

\section{miR-130b-3p suppresses and SIK1 promotes MB tumorigenesis in vivo}

To examine the role of miR-130b-3p and SIK1 in MB tumorigenesis in vivo, we constructed a xenograft animal model by implanting Daoy cells with stable overexpression of miR-130b-3p or knockdown of SIK1 in 6-week-old nude mice. The mice were euthanized after 8 weeks. Tumor volume was significantly smaller in the miR-130b-3p overexpression group (LV16-miR-130b-3p) than in the NC group (LV16-miR-NC). Moreover, tumor weight was also lower in mice inoculated with Daoy-LV16-miR-130b3 p cells compared with those in the control group at week 8 (Fig. 7a-c). Furthermore, miR-130b-3p expression was significantly upregulated by RT-qPCR and FISH (Fig. 7d, e). Similarly, in mice inoculated with sh-SIK1 cells, the average tumor volume was substantially smaller than in sh-GFP mice at week 8 , and the tumor weight was also lower (Fig. $7 \mathrm{f}-\mathrm{h}$ ). Xenografts were also subjected to IHC assays. The results showed that SIK1 expression decreased in the LV16-miR-130b-3p-treated group, similar to the sh-SIK1treated group (Fig. 7i, j). Ki-67 staining of the xenograft tumors was also performed, and the results further confirmed the inhibitory effect of miR-130b-3p and the promotive effect of SIK1 on MB tumorigenesis (Fig. 7i, j).

\section{Discussion}

Exosomes are an important carrier in cell communication $^{10}$. Although exosomes have been studied for several years, the biological roles of exosomal miRNAs are just beginning to be investigated. miRNAs also serve as important intercellular communication tools, as they are transferred between cells via exosomes and influence the phenotypes of their recipient cells ${ }^{26-28}$. In the current study, we previously analyzed the miRNA expression profiles of exosomes derived from MB patient plasma, and found that there was a higher level of miR$130 \mathrm{~b}-3 \mathrm{p}$ in exosomes derived from MB patient plasma than from exosomes derived from healthy control plasma. Exosomal miR-130b-3p from MB patient plasma could be transferred to the $\mathrm{MB}$ cell line and played a tumor suppressor role. miR-130b-3p suppressed MB tumorigenesis by targeting a novel target, SIK1, through the p53 signaling pathways (Fig. 8). Our research provides new insight into the molecular mechanism of $\mathrm{MB}$ and may offer a new therapeutic strategy for MB.
In this research, the expression of miR-130b-3p was higher in circulating exosomes while lower in tumor tissues. This phenomenon was also reported in several other cancers, like lung adenocarcinoma ${ }^{29,30}$, esophagus adenocarcinoma $^{31}$, intestinal-type sinonasal adenocarcinoma ${ }^{32}$, and ovarian cancer ${ }^{33}$. The plausible explanation is exosomes derived from normal cells can act as a carrier for delivery of antitumor factors. But the detailed mechanism is needed for further analysis. To study the origin of the exosomes containing miR-130b-3p, we isolated exosomes from $\mathrm{MB}$ patient plasma and THP-1 culture supernatant that was transfected with miR-130b$3 \mathrm{p}$ mimic, and we found there was higher expression of miR-130b-3p in the isolated exosomes. So we suspected that monocyte in the PBMCs was one source of the exosomes containing miR-130b-3p. We also found that exosomes from THP-1 could also transfer into MB cells, and the expression of miR-130b-3p was higher in cocultured with exosomes derived from THP-1. While this phenomenon disappeared after treatment with GW4869. It was known that monocyte could migrate into tissues and transformed into macrophages ${ }^{34}$. Macrophages were a kind of innate immune cells that played an important role in maintaining homeostasis and host defense $\mathrm{e}^{35}$, especially in the progression from early cancer to metastatic tumor ${ }^{36,37}$. Yin et al. ${ }^{38}$ reported that macrophagederived exosomes played a vital role in the development of tumor. So, the plausible explanation is exosomes derived from mono-macrophage cells can act as a carrier for delivery of antitumor factors. As for how the exosomal miR-130b-3p derived from mono-macrophages, further research is needed.

miR-130b-3p has been shown to have different effects in different cancer types, and functions as an oncogene in thyroid adenomas ${ }^{23}$ and epithelial ovarian cancer ${ }^{39}$. miR130b-3p also acts as a tumor suppressor in breast carcinoma $^{22}$ and ovarian cancer ${ }^{40}$. However, to date, there has been no research concerning the function of miR-130b-3p in $\mathrm{MB}$. In the current study, we have demonstrated that miR-130b-3p played an important suppressive role in the progression of $\mathrm{MB}$. In this study, we found that exosomal miR-130b-3p in MB patient plasma was upregulated than in healthy, but we did not further distinguish whether the expression levels of miR-130b-3p in each molecular subgroup according to current WHO classification was 


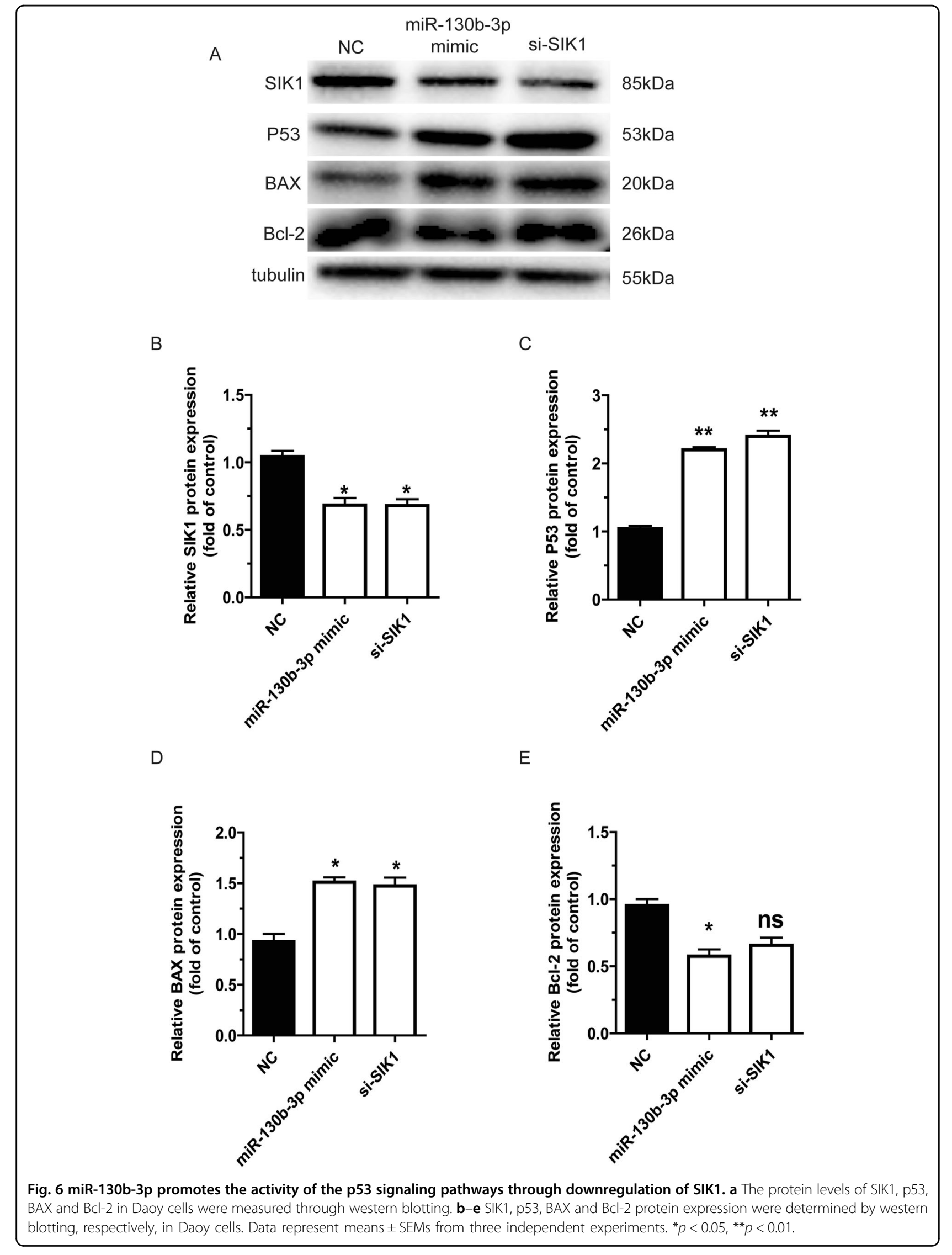




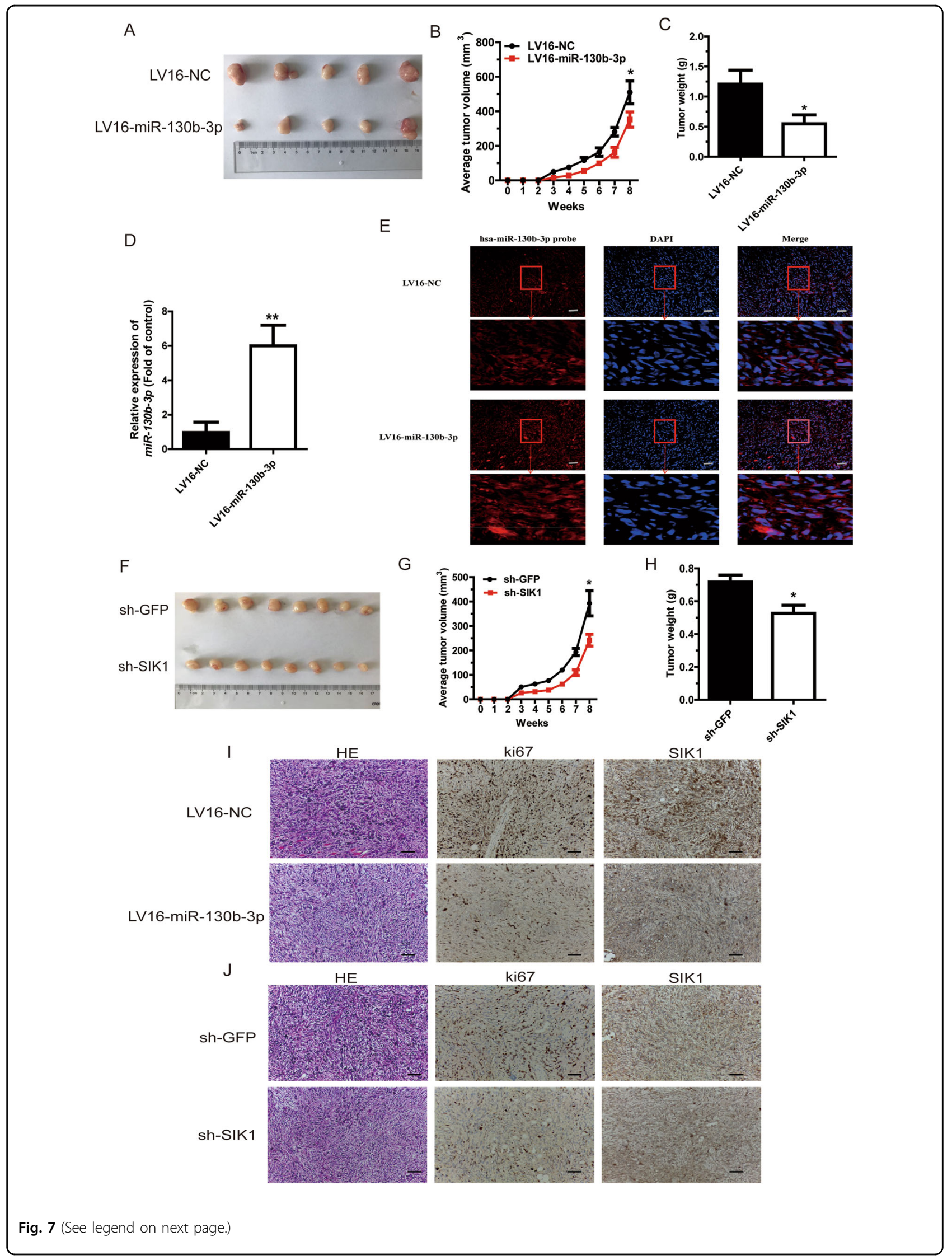


(see figure on previous page)

Fig. 7 miR-130b-3p suppresses and SIK1 promotes MB tumorigenesis in vivo. a Nude mice were injected with subcutaneous xenografts of Daoy cells overexpressing miR-130b-3p or NC and were euthanized after 8 weeks (five mice per group). b Tumor volume was recorded weekly after an injection of Daoy cells transfected with LV16-miR-130b-3p and LV16-NC. c Tumor weight was measured 8 weeks post injection with Daoy cells transfected with LV16-miR-130b-3p and LV16-NC. d, e miR-130b-3p expression was measured in mice tumors by RT-qPCR and FISH. f Mouse tumors were imaged 8 weeks after injection with Daoy cells, and demonstrated decreasing SIK1 expression (eight mice per group). g Tumor volume was recorded weekly after injection with Daoy cells transfected with sh-SIK1 and sh-GFP. $\mathbf{h}$ Tumor weight was measured 8 weeks post injection with Daoy cells transfected with sh-SIK1 or sh-GFP. $\mathbf{i}, \mathbf{j}$ Hematoxylin and eosin (HE) staining (original magnifications $\times 200$ ) of tumors and SIK1 and Ki67 expression in the tumor tissue samples, as determined through IHC. Data represent means \pm SEMs from three independent experiments. ${ }^{*} p<0.05,{ }^{* *} p<0.01$.

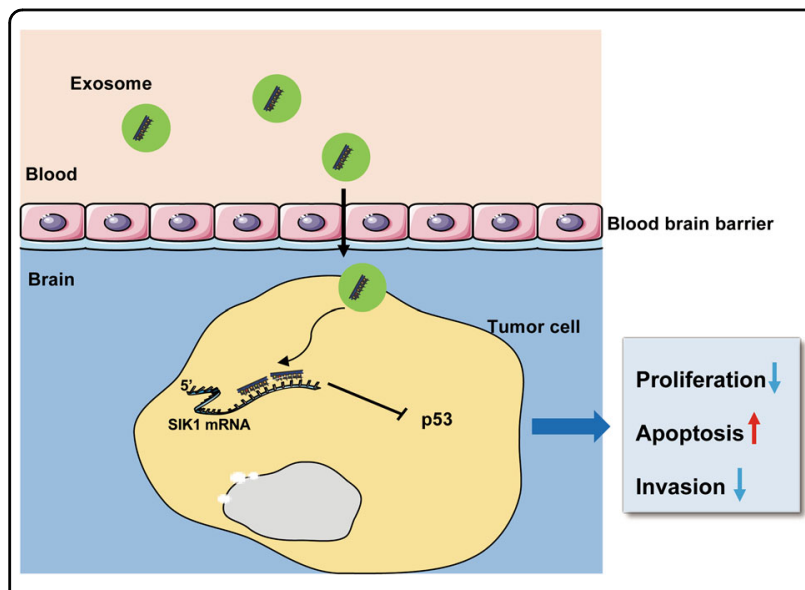

Fig. 8 miR-130b-3p promotes the activity of p53 signaling pathways through downregulation of SIK1. Schematic model of the role of miR-130b-3p in regulating MB tumorigenesis.

different due to the limitation of the number of $\mathrm{MB}$ patients. In the future, a large population of $\mathrm{MB}$ patients is needed to evaluate whether exosomal miR-130b-3p can serve as a biomarker for $\mathrm{MB}$ diagnosis and prognosis.

In this study, we confirmed that SIK1 was an unreported direct target of miR-130b-3p using a dual luciferase reporter assay. SIK1, a serine/threonine kinase, is a member of the AMPK family, which plays vital roles in the regulation of cell growth ${ }^{41}$. Recent evidence has shown that SIK1 performs an important role in human cancers, such as non-small-cell lung cancer ${ }^{42}$, epithelial ovarian cancer $^{43}$ or hepatocellular carcinoma ${ }^{41,44}$. Previous research has shown that SIK1 is a tumor suppressor in many cancers ${ }^{45,46}$. Interestingly, in the current study, we identified SIK1 as a tumor-promoting protein, as it can induce tumor cell proliferation, invasion and metastasis in vivo/vitro. We further found that the overexpression of miR-130b-3p could upregulate the protein expression of p53 and BAX, while downregulating Bcl-2. Therefore, our results indicate that miR-130b-3p may function as a tumor suppressor by inhibiting SIK1 protein expression and subsequent downstream p53 activation in MB cell lines, which might provide a new therapeutic strategy for MB.
In conclusion, the current study demonstrates that the intracellular miR-130b-3p affects the proliferation, migration and invasiveness of $\mathrm{MB}$, which is mediated through targeting SIK1, and exosomal miR-130b-3p play a tumor suppressor role in $\mathrm{MB}$ tumorigenesis. However, further research is needed to understand the more profound molecular mechanism of exosomal miR-130b-3p origin and function in $\mathrm{MB}$ tumorigenesis.

\section{Acknowledgements}

This work was supported by grants from the National Key R\&D Program of China (2016YFC1305102 to Y.Z.), National Natural Science Foundation of China (81671561, 81974248 to Y.Z., 81900751 to X.H.), the International Joint Laboratory Program of National Children's Medical Center (EK1125180109 to Y.Z.), Program for Outstanding Medical Academic Leader (2019LJ19 to Y.Z.) and Shanghai Municipal Planning Commission of Science and Research Fund (201740065 to Y.Z. and 20174 Y0079 to X.H.). Shanghai Pujiang Program (16PJ1401600 to J.F.). Shanghai Committee of Science and Technology (19ZR1406400 to J.F.).

\section{Author details}

'Institute of Pediatrics, Children's Hospital of Fudan University, and the Shanghai Key Laboratory of Medical Epigenetics, International Co-laboratory of Medical Epigenetics and Metabolism, Ministry of Science and Technology, Institutes of Biomedical Sciences, Fudan University, 200032 Shanghai, China. ${ }^{2}$ Department of Neurosurgery, Children's Hospital of Fudan University, Shanghai, China. ${ }^{3} \mathrm{NHC}$ Key Laboratory of Neonatal Diseases, Fudan University, 201102 Shanghai, China

\section{Conflict of interest}

The authors declare that they have no conflict of interest.

\section{Publisher's note}

Springer Nature remains neutral with regard to jurisdictional claims in published maps and institutional affiliations.

Supplementary Information accompanies this paper at (https://doi.org/ 10.1038/s41419-020-2621-y).

Received: 6 January 2020 Revised: 18 May 2020 Accepted: 19 May 2020 Published online: 01 June 2020

\section{References}

1. Ning, M. S., Perkins, S. M., Dewees, T. \& Shinohara, E. T. Evidence of high mortality in long term sunvivors of childhood medulloblastoma. J. NeuroOncol. 122, 321-3-27 (2015).

2. Crawford, J. R., MacDonald, T. J. \& Packer, R. J. Medulloblastoma in childhood: new biological advances. Lancet Neurol. 6, 1073-1085 (2007).

3. Gajjar, A. et al. Risk-adapted craniospinal radiotherapy followed by high-dose chemotherapy and stem-cell rescue in children with newly diagnosed 
medulloblastoma (St Jude Medulloblastoma-96): long-term results from a prospective, multicentre trial. Lancet Oncol. 7, 813-820 (2006).

4. Rutkowski, S. et al. Survival and prognostic factors of early childhood medulloblastoma: an international meta-analysis. J. Clin. Oncol. 28, 4961-4968 (2010).

5. Ellison, D. W. et al. beta-Catenin status predicts a favorable outcome in childhood medulloblastoma: the United Kingdom Children's Cancer Study Group Brain Tumour Committee. J. Clin. Oncol. 23, 7951-7957 (2005).

6. Kumar, V. et al. Challenges and recent advances in medulloblastoma therapy. Trends Pharmacol. Sci. 38, 1061-1084 (2017).

7. Kim, W. et al. The tumor biology and molecular characteristics of medulloblastoma identifying prognostic factors associated with survival outcomes and prognosis. J. Clin. Neurosci. 18, 886-890 (2011).

8. Mathieu, M., Martin-Jaular, L., Lavieu, G. \& Thery, C. Specificities of secretion and uptake of exosomes and other extracellular vesicles for cell-to-cell communication. Nat. Cell Biol. 21, 9-17 (2019).

9. Chopp, M. \& Zhang, Z. G. Emerging potential of exosomes and noncoding microRNAs for the treatment of neurological injury/diseases. Expert Opin. Emerg. Drugs 20, 523-526 (2015).

10. Simons, M. \& Raposo, G. Exosomes-vesicular carriers for intercellular communication. Curr. Opin. Cell Biol. 21, 575-581 (2009).

11. Rodriguez, A., Griffiths-Jones, S., Ashurst, J. L. \& Bradley, A. Identification of mammalian microRNA host genes and transcription units. Genome Res. 14, 1902-1910 (2004).

12. Djuranovic, S., Nahvi, A. \& Green, R. A parsimonious model for gene regulation by miRNAs. Science 331, 550-553 (2011).

13. Bartel, D. P. MicroRNAs: target recognition and regulatory functions. Cell 136, 215-233 (2009).

14. Alexander, M. et al. Exosome-delivered microRNAs modulate the inflammatory response to endotoxin. Nat. Commun. 6, 7321 (2015).

15. Montecalvo, A. et al. Mechanism of transfer of functional microRNAs between mouse dendritic cells via exosomes. Blood 119, 756-766 (2012).

16. Mittelbrunn, M. et al. Unidirectional transfer of microRNA-loaded exosomes from T cells to antigen-presenting cells. Nat. Commun. 2, 282 (2011).

17. Kosaka, N. et al. Neutral sphingomyelinase 2 (nSMase2)-dependent exosomal transfer of angiogenic microRNAs regulate cancer cell metastasis. J. Biol. Chem. 288, 10849-10859 (2013).

18. Egawa, H. et al. The miR-130 family promotes cell migration and invasion in bladder cancer through FAK and Akt phosphorylation by regulating PTEN. Sci. Rep. 6, 20574 (2016)

19. Li, Z. et al. miR-130b targets NKD2 and regulates the Wnt signaling to promote proliferation and inhibit apoptosis in osteosarcoma cells. Biochem. Biophys. Res. Commun. 471, 479-485 (2016)

20. Ye, L., Wang, Y., Nie, L., Qian, S. \& Xu, M. MiR-130 exerts tumor suppressive function on the tumorigenesis of human non-small cell lung cancer by targeting PTEN. Am. J. Transl. Res. 9, 1856-1865 (2017).

21. Yeung, M. L. et al. Roles for microRNAs, miR-93 and miR-130b, and tumor protein 53-induced nuclear protein 1 tumor suppressor in cell growth dysregulation by human T-cell lymphotrophic virus 1. Cancer Res. 68, 8976-8985 (2008).

22. Shui, Y. et al. miR-130b-3p inhibits cell invasion and migration by targeting the Notch ligand Delta-like 1 in breast carcinoma. Gene 609, 80-87 (2017).

23. Leone, V. et al. miR-130b-3p upregulation contributes to the development of thyroid adenomas targeting CCDC6 gene. Eur. Thyroid J. 4, 213-221 (2015).

24. Ismail, N. et al. Macrophage microvesicles induce macrophage differentiation and miR-223 transfer. Blood 121, 984-995 (2013).

25. Holder, B. et al. Macrophage exosomes induce placental inflammatory cytokines: a novel mode of maternal-placental messaging. Traffic 17, 168-178 (2016).
26. Hashimoto, K. et al. Cancer-secreted hsa-miR-940 induces an osteoblastic phenotype in the bone metastatic microenvironment via targeting ARHGAP1 and FAM134A. Proc. Natl Acad. Sci. USA 115, 2204-2209 (2018).

27. Salido-Guadarrama, I., Romero-Cordoba, S., Peralta-Zaragoza, O., Hidalgo-Miranda, A. \& Rodriguez-Dorantes, M. MicroRNAs transported by exosomes in body fluids as mediators of intercellular communication in cancer. OncoTargets Ther. 7, 1327-1338 (2014).

28. Lafourcade, C., Ramirez, J. P., Luarte, A., Fernandez, A. \& Wyneken, U. MiRNAs in astrocyte-derived exosomes as possible mediators of neuronal plasticity. J. Exp. Neurosci. 10, 1-9 (2016)

29. Lawson, J. et al. Selective secretion of microRNAs from lung cancer cells via extracellular vesicles promotes CAMK1D-mediated tube formation in endothelial cells. Oncotarget 8, 83913-83924 (2017).

30. Cancer Genome Atlas Research, N. Comprehensive molecular profiling of lung adenocarcinoma. Nature 511, 543-550 (2014).

31. Warnecke-Eberz, U., Chon, S. H., Holscher, A. H., Drebber, U. \& Bollschweiler, E. Exosomal onco-miRs from serum of patients with adenocarcinoma of the esophagus: comparison of miRNA profiles of exosomes and matching tumor. Tumour Biol. 36, 4643-4653 (2015)

32. Tomasetti, M. et al. MiR-126 in intestinal-type sinonasal adenocarcinomas: exosomal transfer of MiR-126 promotes anti-tumour responses. BMC Cancer 18, 896 (2018).

33. Pan, C. et al. Exosomal microRNAs as tumor markers in epithelial ovarian cancer. Mol. Oncol. 12, 1935-1948 (2018).

34. Yang, J., Zhang, L., Yu, C., Yang, X. F. \& Wang, H. Monocyte and macrophage differentiation: circulation inflammatory monocyte as biomarker for inflammatory diseases. Biomark. Res. 2, 1 (2014).

35. Chanmee, T., Ontong, P., Konno, K. \& Itano, N. Tumor-associated macrophages as major players in the tumor microenvironment. Cancers $\mathbf{6}$, 1670-1690 (2014).

36. Nielsen, S. R. \& Schmid, M. C. Macrophages as key drivers of cancer progression and metastasis. Mediators Inflamm. 2017, 9624760 (2017).

37. Kim, J. \& Bae, J. S. Tumor-associated macrophages and neutrophils in tumor microenvironment. Mediators Inflamm. 2016, 6058147 (2016).

38. Yin, Z. et al. Macrophage-derived exosomal microRNA-501-3p promotes progression of pancreatic ductal adenocarcinoma through the TGFBR3mediated TGF-beta signaling pathway. J. Exp. Clin. Cancer Res. 38, 310 (2019).

39. Zhou, D. et al. Cytidine monophosphate kinase is inhibited by the TGF-beta signalling pathway through the upregulation of miR-130b-3p in human epithelial ovarian cancer. Cell. Signal. 35, 197-207 (2017)

40. Chan, C. K. et al. Tumour-suppressor microRNAs regulate ovarian cancer cell physical properties and invasive behaviour. Open Biol. https://doi.org/10.1098/ rsob.160275 (2016).

41. Qu, C. et al. Salt-inducible Kinase (SIK1) regulates HCC progression and WNT/ beta-catenin activation. J. Hepatol. 64, 1076-1089 (2016).

42. Yao, Y. H. et al. Attenuated LKB1-SIK1 signaling promotes epithelialmesenchymal transition and radioresistance of non-small cell lung cancer cells. Chin. J. Cancer 35, 50 (2016).

43. Hong, B., Zhang, J. \& Yang, W. Activation of the LKB1SIK1 signaling pathway inhibits the TGFbetamediated epithelialmesenchymal transition and apoptosis resistance of ovarian carcinoma cells. Mol. Med. Rep. 17, 2837-2844 (2018).

44. Qu, C. \& Qu, Y. Down-regulation of salt-inducible kinase 1 (SIK1) is mediated by RNF2 in hepatocarcinogenesis. Oncotarget 8, 3144-3155 (2017).

45. Yang, L. et al. SIK1-LNC represses the proliferative, migrative, and invasive abilities of lung cancer cells. OncoTargets Ther. 11, 4197-4206 (2018).

46. Selvik, L. K. et al. Salt-inducible kinase 1 (SIK1) is induced by gastrin and inhibits migration of gastric adenocarcinoma cells. PLOS ONE 9, e112485 (2014). 